\title{
Mobile Interaction for Augmentative and Alternative Communication: a Systematic Mapping
}

\author{
Rúbia E. O. Schultz Ascari \\ Department of Informatics \\ Federal University of Paraná (UFPR) \\ and Technological Federal University \\ of Paraná (UTFPR) \\ Curitiba, Brazil \\ rubia@utfpr.edu.br
}

\author{
Roberto Pereira \\ Department of Informatics \\ Federal University of Paraná (UFPR) \\ Curitiba, Brazil \\ rpereira@inf.ufpr.br
}

\author{
Luciano Silva \\ Department of Informatics \\ Federal University of Paraná (UFPR) \\ Curitiba, Brazil \\ luciano@ufpr.br
}

\begin{abstract}
Verbal communication is essential for socialization, meaning construction and knowledge sharing in a society. When verbal communication does not occur naturally because of constraints in people's and environments capabilities, it is necessary to design alternative means. Augmentative and Alternative Communication (AAC) aims to complement or replace speech to compensate difficulties of verbal expression. AAC systems can provide technological support for people with speech disorders, assisting in the inclusion, learning and sharing of experiences. This paper presents a systematic mapping of the literature to identify research initiatives regarding the use of mobile devices and AAC solutions. The search identified 1366 potentially eligible scientific articles published between 2006 and 2016, indexed by ACM, IEEE, Science Direct, and Springer databases and by the SBC Journal on Interactive Systems. From the retrieved papers, 99 were selected and categorized into themes of research interest: games, autism, usability, assistive technology, AAC, computer interfaces, interaction in mobile devices, education, among others. Most of papers (57 out of 99) presented some form of interaction via mobile devices, and 46 papers were related to assistive technology, from which 14 were related to AAC. The results offer an overview on the applied research on mobile devices for AAC, pointing out to opportunities and challenges in this research domain, with emphasis on the need to promoting the use and effective adoption of assistive technology.
\end{abstract}

Index Terms-Augmentative and Alternative Communication, systematic mapping, mobile devices, interaction.

\section{INTRODUCTION}

Verbal language is the most commonly used medium for people to communicate, yet communication between people can be much more comprehensive. Human beings use nonverbal behavior in order to complete their interpersonal interaction [1]. Communication between people is complemented by several communicative elements (e.g., emotional states, gestures, facial expressions) that allow people to understand each other.

Communication is essential for socialization. When people experience difficulties to express verbally what they intend to communicate, interaction problems arise, hampering learning, family living, social activities, professional practices etc., leading to emotional, social, and cognitive problems [2]. Therefore, alternatives for individuals to communicate to each other and with the world around them are demanded, and computing technology has potential to contribute with interactive and low cost solutions.

Augmentative and Alternative Communication (AAC) aims to complement or replace speech to compensate difficulties of expression by using non-verbal communication systems and intervention strategies [3]. The practice of AAC mediated by computational applications represents a very attractive alternative, mainly by means of mobile devices. Learning opportunities are numerous, and considering the reality of people already connected via smartphones and other mobile devices, creating means to enable everyone's access, anywhere, anytime, can be particularly interesting. AAC can serve as an alternative and effective way to promote social interactions towards a more inclusive and active participation of people in society [4].

The possibilities of using mobile devices for supporting AAC are diverse, interesting and challenging in terms of devices, interface, interaction, social and economic issues. In this article, we present a systematic mapping of the literature to identify research initiatives regarding the use of mobile devices as a tool to improve or facilitate the communication of people with some type of communication disability.

\section{Augmentative And Alternative Communication}

Augmentative and Alternative Communication refers to all forms of communication that can complement or replace speech. AAC covers the needs of reception, understanding and verbal expression, increasing the communicative interaction of individuals without orality. "Augmentative" communication systems complement oral language when it is not possible to communicate effectively with other people and the environment. "Alternative" communication systems replace oral language when it cannot be understood or has been lost. Both types of systems support people with communication problems to interact with other people, expressing their opinions, feelings and making personal decisions to lead and control their lives [5].

Non-speakers usually use AAC technology to enhance their communication (non-verbal gestures and non-lexical sounds, such as laughter) as well as an alternative to oral discourse [6]. 
The very aim of AAC is to enable all people to communicate, by strengthening ties with the environment in which they are inserted, with their families and with their peers.

AAC devices range from low-tech devices, such as photo cards and communication boards, to high-tech versions such as electronic communication boards, computerized voice synthesizers and specific software. For people with expressive language impairment (e.g., resulting from autism, cerebral palsy), these devices provide tools that allow the selection of words, symbols and images to communicate their thoughts, intentions and conversation with others by means of digitized or synthesized voice [6].

In the past, there may have been criticism about the effective usefulness of AAC solutions. Some argued that AAC affects learning negatively as an individual could prefer to use it than developing the necessary capabilities [7]. Currently, it is generally agreed that AAC is essential for the development of individuals with communication difficulties, assisting individuals with intellectual disabilities in their learning process [8].

\section{A. AAC and Mobile Computing}

In some contexts, devices with touch screens are more effective than pointers (e.g., a mouse) to allow interaction with computers. Computer games are becoming more and more mediated by means of devices like tablets, smartphones and sensors. Mobile computing applications have been often presented as assistive technology for children with special needs. These applications seek to assist children in different contexts, such as learning, reading and developing everyday skills [9].

The developments in mobile computing and advancements in electronic communication aids for nonspeaking individuals are inherently intertwined through the history of their research, development, commercialization, use, and reuse [6]. Individuals with various disabilities need to be recovered from and rewritten into the history of how communication technology are designed, marketed, and adopted [6].

As the use of mobile devices has gained popularity, there are applications being developed to promote learning in several areas of knowledge, such as: mobile applications to assist people with visual impairment to communicate and control an Android mobile phone via speech recognition [10]; serious game to teach first aid for individuals with autism spectrum disorder (ASD) [11]; collaborative games, such as the ComFiM (communication through the exchange of figures for multi-touch devices), a game to generate communicative situations among children with autism in a collaborative environment [12]; alternative communication systems for web and mobile devices to support alternative communication for inclusion processes to autism [13], to cite a few.

AAC supported by mobile devices can increase the autonomy of people with special communication needs. Because mobiles are well-disseminated, have high processing and memory capacity, and are relatively easy to acquire, such devices become an interesting alternative compared to computers or other equipment dedicated to provide the use of an AAC system.

\section{Systematic MAPPING}

A systematic mapping review allows to identify, analyze and work on available research relevant to a particular research question, a topic area, or a phenomenon of interest [14]. In a systematic review, the search process is conducted according to a well-defined sequence of steps, following a previously planned study protocol [15].

Systematic mappings are a particular type of systematic review with a broader scope, designed to cover and give an overview of a research area by classifying and counting contributions according to pre-defined categories [16] [17]. A systematic mapping studies the literature to identify what topics are being covered and how, where research have been published and by whom, what are the most common practices and tools, the gaps and opportunities and so on.

While systematic literature reviews focus on gathering and synthesizing evidence, considering the evidence strength, systematic literature mapping are primarily used to structure a research area [17]. This article presents a systematic mapping of literature on studies related to AAC, mapping studies that investigate mobile devices as assistive technology solutions to enable or improve the communication capacities of people who have some type of expression or interaction difficulty. The mapping considered scientific articles/papers indexed by ACM, IEEE, Science Direct, Springer databases and by the SBC Journal on Interactive Systems, published between 2006 and 2016.

The plan for the systematic mapping was developed according to the guidelines from Munzlinger et al. [15] and Petersen et al. [17]. The first step was to plan and formalize the study protocol, specifying the research problem, its objective, the research questions and the keywords. Tab. I presents details about the study protocol.

TABLE I

STUDY PLAN

\begin{tabular}{l}
\hline Research problem: To investigate modalities of interaction on mobile \\
devices able to be applied as assistive technology for AAC. \\
\hline Objective: To map research possibilities in assistive technology in mobile \\
devices for supporting AAC. \\
\hline General question: What are the recent theoretical solutions or practical \\
tools for supporting AAC via mobile devices, and what modalities of \\
interaction have been employed? \\
\hline Research questions: \\
1) What are the recent modalities of interaction used on mobile devices? \\
2) What are the interactive limitations of existing studies aimed at the \\
practice of AAC via mobile devices? \\
3) Are there specific methodologies to stimulate AAC practice in mobile \\
devices? What modes of interaction do they employ? \\
4) What kind of user interfaces are used to facilitate interaction via mobile \\
devices? \\
5) What are the aspects of Human-Computer Interaction being considered? \\
How? \\
6) What applications are used as assistive technology for AAC in mobile \\
devices?
\end{tabular}

For the study protocol, selection criteria were defined and applied as a first filter for retrieved studies. Selection criteria 
were divided into inclusion and exclusion criteria and applied to classify the studies according to their metadata (title, abstract and keywords). Studies that met at least one of the inclusion criteria were included, and studies that felt in at least one of the exclusion criteria were excluded. Tab. II presents the inclusion and exclusion criteria of the first filter.

TABLE II

INCLUSION AND EXCLUSION CRITERIA FOR THE 1ST FILTER

\begin{tabular}{|l|l|}
\hline \multicolumn{2}{|c|}{ 1st Filter } \\
\hline Inclusion Criteria & Exclusion Criteria \\
\hline $\begin{array}{l}\text { IC1: The study defines or presents } \\
\text { instruments for AAC in mobile de- } \\
\text { vices. }\end{array}$ & $\begin{array}{l}\text { EC1: The study presents no evalu- } \\
\text { ation or analysis of applications for } \\
\text { AAC. }\end{array}$ \\
\hline $\begin{array}{l}\text { IC2: The study investigates, com- } \\
\text { pares or evaluates AAC applica- }\end{array}$ & $\begin{array}{l}\text { EC2: The study mentions AAC via } \\
\text { mobile devices only as proposal for } \\
\text { tions for mobile devices. }\end{array}$ \\
\hline $\begin{array}{l}\text { IC3: The study defines or presents } \\
\text { fifferent modalities of interaction }\end{array}$ & $\begin{array}{l}\text { EC3: The study was not related to } \\
\text { AAC or modalities of interaction }\end{array}$ \\
via mobile devices. & via mobile devices. \\
\hline $\begin{array}{l}\text { IC4: The study presents the appli- } \\
\text { cation of a methodology for prac- } \\
\text { ticing AAC or employing assistive } \\
\text { technology in mobile devices. }\end{array}$ & $\begin{array}{l}\text { ECr: The study was published be- } \\
\text { fore 2006. }\end{array}$ \\
\hline
\end{tabular}

The selection criteria for the second filter were defined and applied on the complete reading of studies resulting from the first filter. Tab. III describes the criteria.

In the second step of the mapping process search expressions were defined, calibrated and adaptation for each selected database. The searches were carried out in November 2016, returning 1366 studies: 1217 were excluded by the first filter, and 50 were excluded by the second filter, resulting in a set of 99 studies. Tab. IV presents an overview of the selection process.

TABLE III

SELECTION CRITERIA FOR THE 2ND FILTER

\begin{tabular}{|l|}
\hline \multicolumn{1}{|c|}{ 2nd Filter - Selection Criteria } \\
\hline MOB: Application running on mobile devices or related theme. \\
\hline AAC: Application for AAC or related topic. \\
\hline ASS: Informations about accessibility or assistive technology. \\
\hline $\begin{array}{l}\text { INT: Informations about computational interfaces or interaction in mobile } \\
\text { devices. }\end{array}$ \\
\hline $\begin{array}{l}\text { HCI: Information on topics from Human-Computer Interaction in mobile } \\
\text { devices. }\end{array}$ \\
\hline
\end{tabular}

Tab. V shows the data extraction form used to standardize the data extracted from the publications read, aiming to reduce the bias of the results and the informality of the process. The extraction was performed by the first author and reviewed by the second author by tracing back the information in the extraction form to the statements in each paper, and checking their correctness. Categories were created dynamically as data were extracted in order to reflect the data set resulting from the extraction process itself. A dynamic scheme was adopted instead of a predefined scheme in order to reflect the extracted content. On the one hand, a predefined categorization can represent a more structured categorization; on the other hand,
TABLE IV

SEARCH EXPRESSION AND RESULTS OBTAINED

\section{Example of search expression}

TITLE(mobile OR "alternative communication" OR "augmentative and alternative communication" OR "AAC") AND (interact OR touch OR gyroscope OR accelerometer OR vibracall OR tablet OR iPad OR phone) AND (ABSTRACT(mobile AND communication AND interact) OR ABSTRACT(mobile AND communication AND disability)).

\begin{tabular}{|l|l|l|l|}
\hline Database & $\begin{array}{l}\text { Number of } \\
\text { studies }\end{array}$ & 1st filter & 2nd filter \\
\hline ACM & 405 & 57 & 37 \\
\hline IEEE & 835 & 62 & 37 \\
\hline Science Direct & 108 & 13 & 10 \\
\hline Springer & 16 & 15 & 13 \\
\hline $\begin{array}{l}\text { SBC Journal on Interactive } \\
\text { Systems }\end{array}$ & 2 & 2 & 2 \\
\hline Total of selected studies & $\mathbf{1 3 6 6}$ & $\mathbf{1 4 9}$ & $\mathbf{9 9}$ \\
\hline
\end{tabular}

it prevents the identification of other relevant categories to represent the selected set. Because the nature of this study is an open and comprehensive mapping, we opted for this more flexible form of classification, generated from the data extraction form used.

TABLE V

DATA EXTRACTION FORM

\begin{tabular}{|l|}
\hline Attributes \\
\hline Article title \\
\hline Year of publication \\
\hline Name (s) of the author (s) \\
\hline Author's institution country (s) \\
\hline Source of publication \\
\hline Involved technology \\
\hline Brief description \\
\hline $\begin{array}{l}\text { Contribution area (mobile interaction, mobile computing interface, AAC, } \\
\text { assistive technology, autism, games, usability, education or other). }\end{array}$ \\
\hline $\begin{array}{l}\text { Type of contribution (theoretical, systematic review of literature, system- } \\
\text { atic mapping, survey, application, model, method, technique, comparison). }\end{array}$ \\
\hline Database used in the search: name, public / private. \\
\hline
\end{tabular}

\section{A. Threats to the validity of the study}

This section discusses the threats to validity that might have affected the results of this systematic mapping. The review protocol was validated to ensure that the research was as correct, complete and objective as possible. However, possible limitations in two moments of the review process were identified: in the publication selection and in the data extraction.

Different threats to validity can be pointed out in this study. One of the threats is missing relevant studies in the area. In fact, we cannot guarantee that all related papers published are included in this mapping. The search for publications was performed only on a limited set of journals and databases, assuming that these search engines tend to contain the majority of the relevant studies. The terms used in search strings may have many synonyms and the search string itself narrows the possible results. Although we mitigate this threat as much as possible by following the references in the primary studies, 
we recognize that relevant papers are not included because of divergence in the use of terms and expressions.

As another threat, it is possible that some kind of inaccuracy or misclassification has occurred in the data extraction performed in this systematic mapping, mainly because the data extraction was done individually by a researcher. The coherence of our classification scheme can also introduce bias to the data analysis, and other researchers may possibly come up with different classification schemes. To reduce these threats, data extraction and classification were conducted by the first author and validated by the second. Disagreements were resolved by means of discussions or led to the refinement of the classification scheme, leading sometimes, to the reclassification and new validation of previously classified publications. This procedure was repeated until there were no disagreements.

Petersen et al. [17] reviewed existing validity classification schemes and discussed their applicability to software engineering. Based on the study of these authors, the following types of validity where taken into account to minimize the threats to the validity of the study: descriptive validity, theoretical validity, generalizability, interpretive validity.

Descriptive validity is the extent to which observations are described accurately and objectively [17]. To reduce this threat, a data extraction form has been designed to support the recording of data. The form objectified the data extraction process and could always be revisited. Hence, this threat is considered as being under control.

Theoretical validity is determined by our ability of being able to capture what we intend to capture [17]. To reduce this threat, the set of research questions was evaluated by the first author and later by the second and third author. The first and second filters were applied, and the remaining articles were read in full, making possible the extraction of answers to these questions.

Generalizability refers to how much it is possible to do a research and generalize the results to come from the proposed research process. Petersen et al. [17] presented a distinction between external generalizability (generalizability between groups or organizations) and internal generalizability (generalization within a group). To avoid the threat of external generalizability an own and theoretically advocated protocol [17] was used, and to avoid internal generalizability were defined research questions and own inclusion / exclusion criteria that allow the expansion or reproduction of the research in a different period, guaranteeing the generalizability of the study.

Interpretive validity is achieved when the conclusions drawn are reasonable given the data, and hence maps to conclusion validity [17]. A threat in interpreting the data is researcher bias, which is minimized through the review process carried out by the authors.

The repeatability requires detailed reporting of the research process [17]. We reported the systematic mapping process followed, and also elaborated on actions taken to reduce possible threats to validity.

\section{RESUlts}

After selecting and reading, studies were categorized by publication year (Fig. 1). The majority of studies (57 of 99) was published in the last 4 years, suggesting the research topic has received attention from the academy and evolved.

Considering the authors' institutions and their respective countries (Fig. 2), most of studies have been published by authors working in institutions from the United States, Korea, China and Germany.

Selected studies were categorized into themes of research interest: games, autism, usability, assistive technology, AAC, computer interfaces, interaction in mobile devices, education, or others. Some studies have been categorized into more than one theme. Results are presented in Fig. 3. Although studies on varied themes related to mobile technology were found, studies focused on assistive technology, education, $\mathrm{AAC}$, computational interfaces and interaction associated with mobile devices were quite representative, and are strongly related to the main objective of this study.

The categorization scheme was created and reviewed while data extraction took place. When extracting data from a specific paper, the first author tried to categorize the paper into an existing category. If the paper did not fit into any existing category, then a new category was created. Naturally, categories evolved during all the extraction process (e.g., categories were merged and refined as needed). Once data was extracted, the categorization scheme was reviewed by the other authors and eventual adjustments were made.

The categorization of the selected studies and their corresponding references are available in Tab. VI. Some selected studies are detailed below according to the categorization scheme developed in this study.

TABLE VI

STUDIES CATEGORIZED BY SUBJECT

\begin{tabular}{|l|l|}
\hline Subject & Studies \\
\hline AAC & {$[6][11][18][19][20][21][22][23][24][25]$} \\
& {$[26][27][28][29]$} \\
\hline Assistive technology & {$[6][9][10][11][13][18][19][20][21][22]$} \\
& {$[23][24][25][26][27][28][29][30][31][32]$} \\
& {$[33][34][35][36][37][38][39][40][41][42]$} \\
& {$[43][44][45][46][47][48][49][50][51][52]$} \\
& {$[53][54][55][56][57][58]$} \\
\hline Autism & {$[10][23][24][28][44][53]$} \\
\hline Computer Interfaces & {$[13][36][37][38][41][42][43][59][60]$} \\
& {$[61][62][63][64][65][66][67][68]$} \\
\hline Education, teaching & {$[23][28][29][35][37][44][46][47][58]$} \\
and learning & {$[64][69][70][71][72]$} \\
\hline Games & {$[51][59][70][71][73][74][75]$} \\
\hline Mobile Interaction & {$[12][13][18][19][20][30][31][32][33]$} \\
& {$[34][35][36][37][38][39][40][41][42]$} \\
& {$[59][60][61][62][63][64][69][70][73]$} \\
& {$[74][76][77][78][79][80][81][82][83]$} \\
& {$[84][85][86][87][88][89][90][91][92]$} \\
& {$[93][94][95][96][97][98][99][100][101]$} \\
& {$[102][103][104][105][106][107]$} \\
\hline Usability & {$[12][38][42][60][93][105][108][109]$} \\
\hline
\end{tabular}




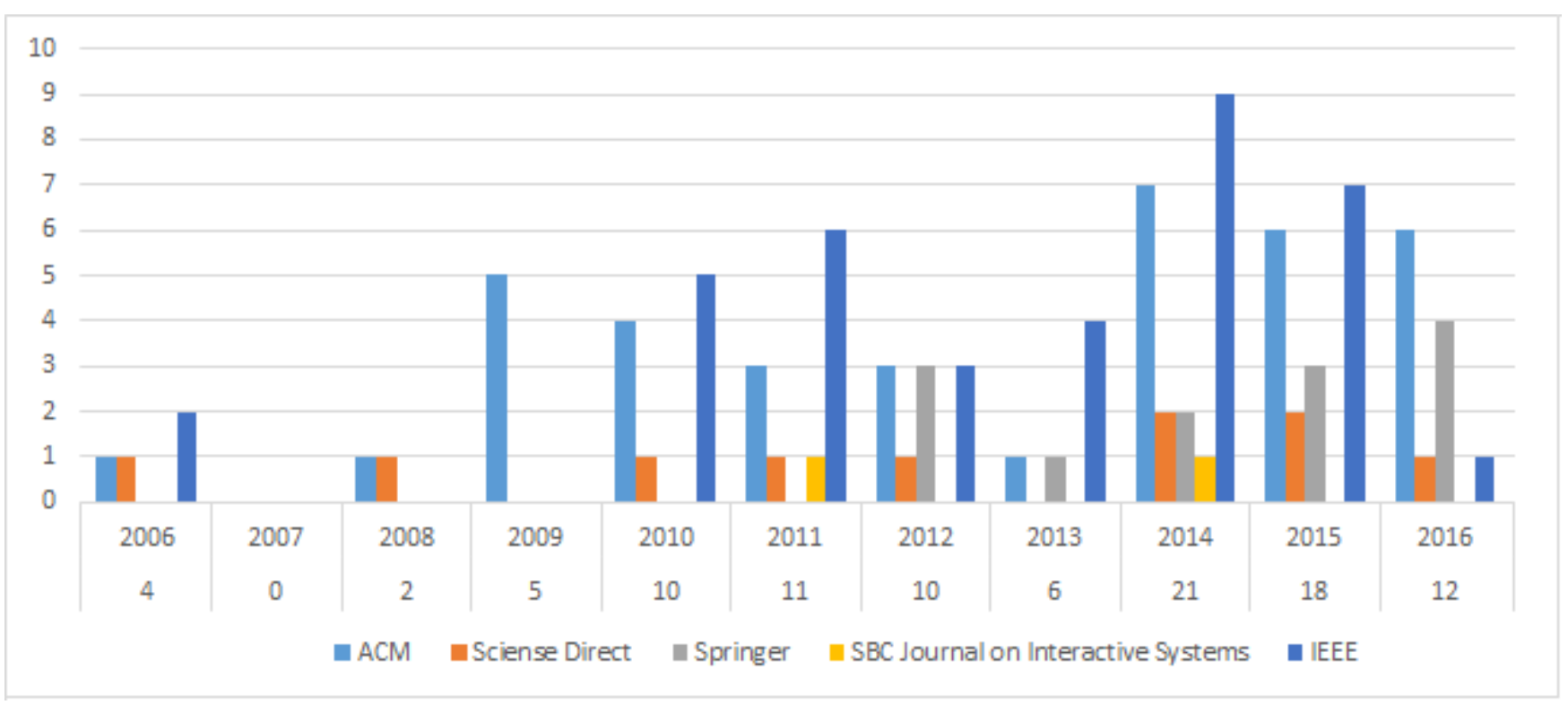

Fig. 1. Publications categorized by year.

\section{A. Assistive technology}

From the studies categorized as assistive technology, some were related to communication or interaction disorders. Studies in this area generally present initiatives to assist or investigate a specific target audience. Some examples are children with severe speech and physical impairment; people with motor difficulties; quadriplegic people; people with intellectual and development disabilities; elderly people with varying degrees of dementia or suffering from chronic diseases; people with speech, hearing or visual impairment; children with learning difficulties (dyslexia); children with cognitive and spastic disabilities; children with Autistic Spectrum Disorder; people with various communication difficulties caused by cerebral palsy, Parkinson disease, Down syndrome, among others. Other examples are students with disability; individuals with aphasia (disruption of the formulation and understanding of the language); and people with deficit of prosody (relative to the good pronunciation of the words).

\section{B. $A A C$}

The mapped studies investigate assistive technology via mobile devices and present challenges to be overcome. For instance, Moffatt et al. [11] cite as future study to: keep focus on communication, not technology; develop innovative approaches to service delivery for AAC; ensure easy access for individuals requiring $\mathrm{AAC}$; and to improve $\mathrm{AAC}$ solutions to support a wide variety of communication functions.

Tab. VII presents some examples of studies that deal specifically with applications for AAC, whether proposing a new application or evaluating existing applications.

The cited studies present solutions aimed at supporting people with communication difficulties to express themselves, to be understood and to perform routine activities in a more facilitated way. Habitually AAC systems such as cited in Babic
TABLE VII

EXAMPLES OF PAPERS FOCUSING ON AAC

\begin{tabular}{|c|l|}
\hline 1 & $\begin{array}{l}\text { Development and evaluation of a mobile application for a personal } \\
\text { narrative system to children with severe speech and physical impair- } \\
\text { ment. [21] }\end{array}$ \\
\hline 2 & $\begin{array}{l}\text { Application for deaf people, people with language disorders, or non- } \\
\text { native language users to report emergencies by means of icons or } \\
\text { pictograms. [22] }\end{array}$ \\
\hline 3 & $\begin{array}{l}\text { Design and implementation of a mobile interface using an input } \\
\text { device via Morse code. [19] }\end{array}$ \\
\hline 4 & $\begin{array}{l}\text { Application to support tutors who employ applied behavior analysis } \\
\text { in people with Autistic Spectrum Disorder, based on AAC and } \\
\text { discrete trial training (method of teaching in simplified and structured } \\
\text { steps). [23] }\end{array}$ \\
\hline 5 & $\begin{array}{l}\text { Study about assistive technology and how they can provide greater } \\
\text { independence and integration of their users with the community. [24] }\end{array}$ \\
\hline 6 & $\begin{array}{l}\text { Application that allows direct communication via voice and SMS, } \\
\text { allowing control of smartphones and home appliances using NFC } \\
\text { (Near Field communication), a wireless technology that allows the } \\
\text { exchange of information between compatible devices close of each } \\
\text { other. [18] }\end{array}$ \\
\hline 7 & $\begin{array}{l}\text { Evaluation of high technology AAC devices and their use by } \\
\text { individuals with aphasia. [11] }\end{array}$ \\
\hline 8 & $\begin{array}{l}\text { Application that allows to create sentences from (i) embedded } \\
\text { predefined symbols in the application or (ii) new user generated } \\
\text { symbols added manually to the application using a symbol editor. } \\
\text { [29] }\end{array}$ \\
\hline 9 & $\begin{array}{l}\text { Device designed to assist deaf-blind individuals to communicate by } \\
\text { means of an intelligent glove that translates the Braille alphabet into } \\
\text { text, and vice versa, and communicates the message via SMS to a } \\
\text { remote contact. [37] }\end{array}$ \\
\hline 10 & $\begin{array}{l}\text { Project for developing an AAC cloud system, adopted in classroom } \\
\text { for teaching and learning for children with ASD in Hong Kong. [28] }\end{array}$ \\
\hline 11 & $\begin{array}{l}\text { A software development process model for implementing AAC } \\
\text { applications, which suggests some specific principles to be followed } \\
\text { to successfully implement accessibility features. [11] }\end{array}$ \\
\hline 12 & $\begin{array}{l}\text { Mobile application for children between 3 and 12 years with alterna- } \\
\text { tive communication function and reading tool that shows the spelling } \\
\text { of the word being heard. [25] }\end{array}$ \\
\hline
\end{tabular}




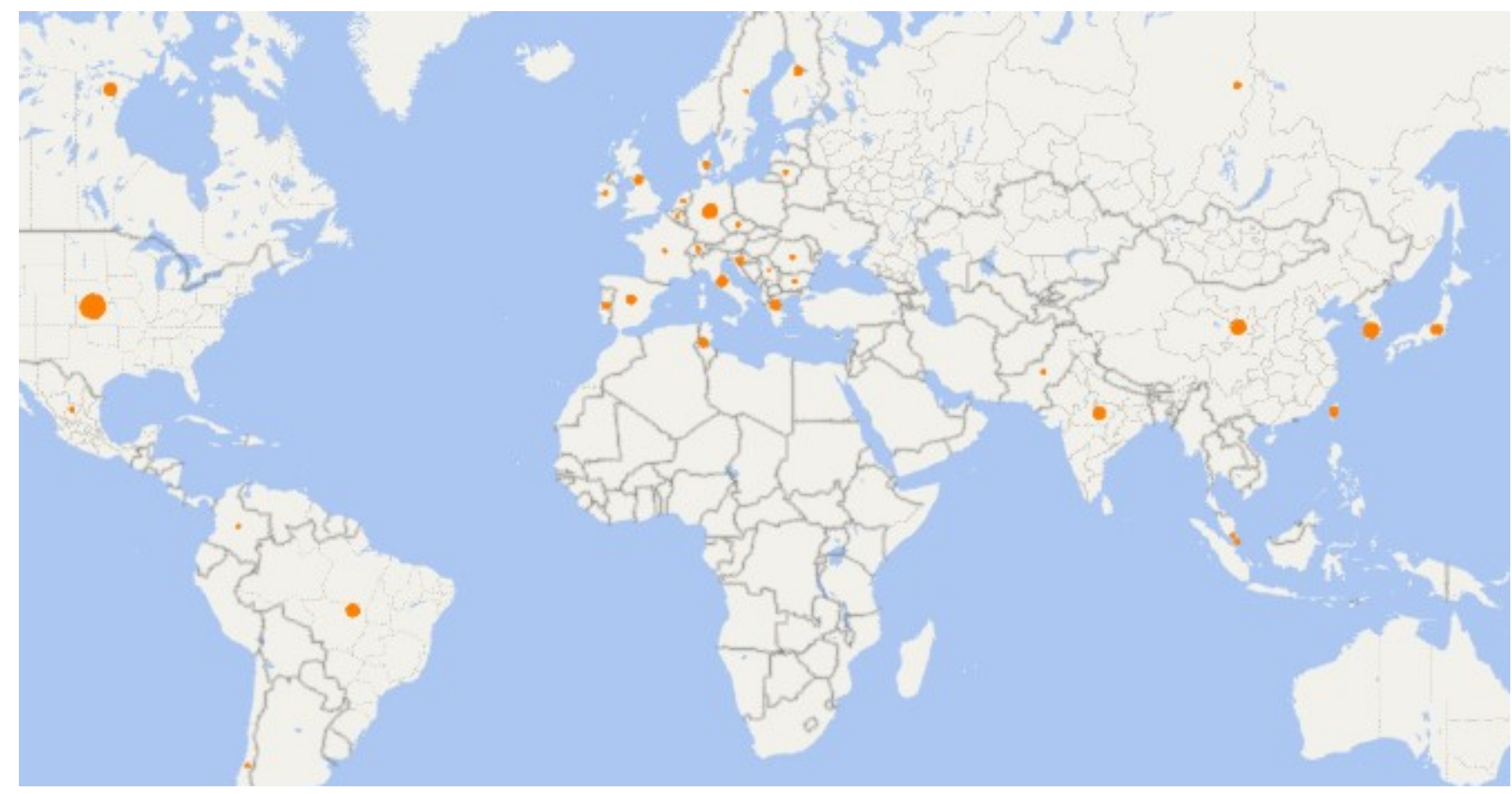

Fig. 2. Publications categorized according to the country of author's institution.

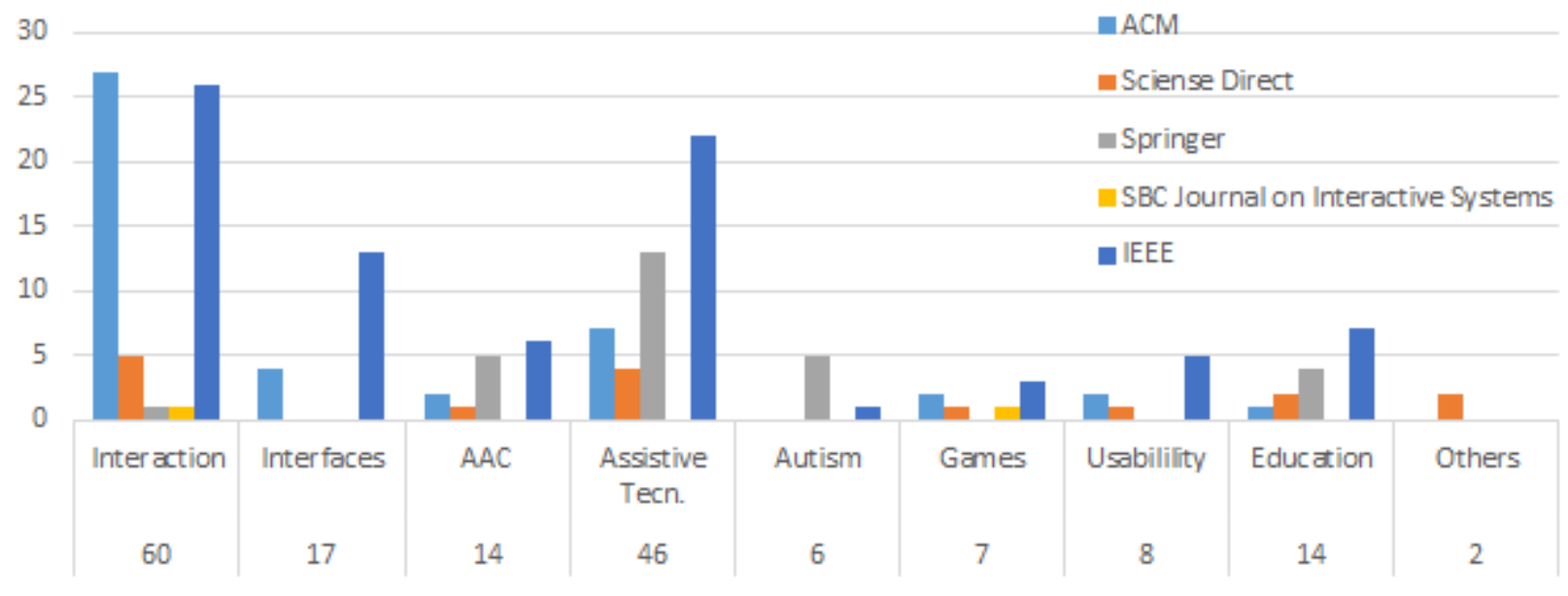

Fig. 3. Publications categorized by subject.

et al. [29], are based on the use of symbols, represented in the form of user-created icons, or in the form of pictograms used in traditional AAC systems. Voice output communication aids and speech generating devices have also been combined with new technology to generate more efficient systems.

Tab. VIII presents some devices and applications for AAC cited in the publications read, followed by some characteristics obtained directly from the publications and on websites of suppliers or manufacturers.

\section{Mobile computing interfaces}

Tab. IX presents some interfaces for use in mobile devices. The described studies are intend to increase user experience on mobile devices by moving from traditional touch interaction to more accessible and easier to use interfaces.

\section{Education, teaching and learning}

Individuals with communication / interaction disorders may present learning difficulties and / or interaction problems in the school environment. Although not the main focus of the mapping, it was possible to identify in the read studies, some research that approach this subject (Tab. X), even if they are not directly related to the AAC theme.

AAC can improve communication and increase children vocabulary knowledge [110]. It has also shown positive effects and can improve interactions with typically developing 
TABLE VIII

EXAMPLES OF APPLICATIONS AND DEVICES FOR AAC

\begin{tabular}{|c|c|}
\hline (Cited in & $\begin{array}{l}\text { pplication that allows people with aphasia to capture and manage digital photographs to support face-to-face } \\
\text { ommunication. }\end{array}$ \\
\hline $\begin{array}{l}\text { Komunikator }+(\text { Cited } \\
\text { in [29]) }\end{array}$ & $\begin{array}{l}\text { pplication that allows to combine phrases and sentences using symbols that can be captured by an integrated camera, } \\
\text { evice image gallery, or symbols from three non-commercial galleries (ARAASAC, Mulberry, and Scler). }\end{array}$ \\
\hline GoTalk (Cited in [23]) & troductory recording communication device which is useful for beginning augmentative communicators. \\
\hline $\begin{array}{l}\text { Activity Pad (Cited in } \\
{[23] \text { ) }}\end{array}$ & $\begin{array}{l}\text { Communication aid with a microchip so different layouts can be personalised for different contexts. Uses voice output, } \\
\text { visual stimulation and tactile activation. }\end{array}$ \\
\hline $\begin{array}{l}\text { Dynavox (Cited in } \\
[23])\end{array}$ & $\begin{array}{l}\text { Speech generating device that creates a spoken message from a picture that is tapped by the user or a message that is } \\
\text { typed into the keyboard, but can also track eye movement and puffs of air. }\end{array}$ \\
\hline $\begin{array}{l}\text { Proloquo2Go (Cited in } \\
\text { [11] [21] [24]) }\end{array}$ & $\begin{array}{l}\text { Application that provides natural sounding text-to-speech voices, high resolution up-to-date symbols, automatic } \\
\text { conjugations, a default vocabulary, word prediction, full expandability. }\end{array}$ \\
\hline $\begin{array}{l}\text { TapToTalk (Cited in } \\
\text { [25] [47]) }\end{array}$ & $\begin{array}{l}\text { Application that renders smartphones and tablets into AAC devices. It helps these children communicate and interact } \\
\text { with their mobile gadgets by tapping pictures and repeating what is said. }\end{array}$ \\
\hline $\begin{array}{l}\text { Lingraphica (Cited in } \\
[11][28])\end{array}$ & $\begin{array}{l}\text { Speech-generating device that exploring familiar scenes to practice words and build functional communication messages. } \\
\text { Use of symbols in storyboarding. }\end{array}$ \\
\hline Tango (Cited in [23]) & $\begin{array}{l}\text { ommunication aid that allows you to record the actual voice and actions through video to be relayed to the } \\
\text { ommunication partner. It helps in understanding the emotional context of a message. }\end{array}$ \\
\hline $\begin{array}{l}\text { AutisMate (Cited in } \\
[24])\end{array}$ & $\begin{array}{l}\text { Application that enables the user to personalize the content, applying it to relevant skills and life experiences that users } \\
\text { need to learn. Uses a hybrid approach of grid-based and visual scene display technology. }\end{array}$ \\
\hline $\begin{array}{l}\text { DynaVoxXpress (Cited } \\
\text { in [18]) }\end{array}$ & $\begin{array}{l}\text { Handheld augmentative communication device, it delivers a full range of communication capabilities and offers optional } \\
\text { web capabilities for surfing the Internet, copying images from web pages and sending email. }\end{array}$ \\
\hline $\begin{array}{l}\text { Gateway (Cited in } \\
[18])\end{array}$ & $\begin{array}{l}\text { Research-based core word vocabulary designed for users of AAC systems. It includes both text and symbol-based } \\
\text { vocabularies that are combined with spelling and word prediction to accommodate the needs of both literate and } \\
\text { non-literate users. }\end{array}$ \\
\hline $\begin{array}{l}\text { MinSpeak (Cited in } \\
[18])\end{array}$ & $\begin{array}{l}\text { emantic compaction system in that pictures take on multiple meanings, which when linked together in short sequences, } \\
\text { eate words, phrases and sentences. }\end{array}$ \\
\hline $\begin{array}{l}\text { Tellus Smart (Cited in } \\
[11])\end{array}$ & ded Communication Aid software. \\
\hline Vantage (Cited in [11]) & $\begin{array}{l}\text { Is a touch screen for access, and can compose message through combining icons and use of the keyboard. It is a } \\
\text { medium weight device, but portable. }\end{array}$ \\
\hline $\begin{array}{l}\text { Gus Communicator } \\
\text { (Cited in }[11])\end{array}$ & road range of tablet based speech packages. \\
\hline $\begin{array}{l}\text { TalkRocketGo (Cited } \\
\text { in [11]) }\end{array}$ & $\begin{array}{l}\text { A mobile AAC device for iOS and Android that helps people with Autism, Crebral Palsy, Stroke, Traumatic Brain } \\
\text { Injury, Parkinson's (and others) speak out loud. }\end{array}$ \\
\hline $\begin{array}{l}\text { SmallTalk (Cited in } \\
[11])\end{array}$ & $\begin{array}{l}\text { Application that provides a vocabulary of pictures and videos that talk in a natural human voice. It allows to personalize } \\
\text { and expand the vocabulary by using Lingraphica. Also contains mouth-position videos for practice and self-cuing. }\end{array}$ \\
\hline $\begin{array}{l}\text { VocaBeans (Cited in } \\
[11])\end{array}$ & $\begin{array}{l}\text { Helps people with speech conditions to communicate. Each VocaBean is a picture and sound representing a word or } \\
\text { phrase. }\end{array}$ \\
\hline $\begin{array}{l}\text { SentenceShaper (Cited } \\
\text { in [11]) }\end{array}$ & $\begin{array}{l}\text { Communication system designed to allow people with aphasia to create sentences and even narratives in their own } \\
\text { voices. It can be used for both communication assistance and language therapy. }\end{array}$ \\
\hline $\begin{array}{l}\text { TalksBac } \quad \text { (Cited in } \\
[11])\end{array}$ & $\begin{array}{l}\text { AAC system word-based and exploits the ability of some nonfluent individuals with aphasia to recognize familiar words } \\
\text { and short sentences. }\end{array}$ \\
\hline PROSE (Cited in [11]) & $\begin{array}{l}\text { A gesture to speech AAC app based on a social construct of conversation. Supported interactive storytelling, allowing } \\
\text { users to control the narration of a story instead of delivering a monologue. }\end{array}$ \\
\hline XTag (Cited in [11]) & $\begin{array}{l}\text { pports the retelling of past experiences via a tagging and sharing application that couples picture taking with extra } \\
\text { ormation such as mood and location. }\end{array}$ \\
\hline $\begin{array}{l}\text { Camelandar (Cited in } \\
[11])\end{array}$ & vides a structure for organizing and sharing these daily life stories. \\
\hline $\begin{array}{l}\text { Storytelling } \\
\text { application } \\
\text { in }[11])\end{array}$ & $\begin{array}{l}\text { or individuals with expressive aphasia, application that supported social exchanges through a multi-modal tablet-based } \\
\text { terface that supported taking photographs, making drawings and annotations, and recording sounds. }\end{array}$ \\
\hline
\end{tabular}

peers and consequently social communication [111]. However, practitioners still face challenges in deploying new technology in the classroom [112]. AAC requires a multidisciplinary approach [113]. According to Light et al. [114], to truly harness the power of technology, rehabilitation and education professionals must ensure that AAC intervention is directed not by the devices but by the individual's communication needs.

\section{E. Mobile interaction}

Most of studies resulting from the map presented some form of interaction in mobile devices: 57 publications were categorized according to the main theme and are presented in Fig. 4. These interaction modalities represent possible ways of interacting with mobile devices and can be combined to explore the concept of multimodal interaction in AAC systems.

It has been found that interactive, multi-sensory interaction and the integration of different technology present great potential to improve the user experience on mobile devices.

\section{F. Games, Autism and Usability}

It was noticed that multimodal interaction on mobile devices can be explored in several ways as assistive technology: Cakic et al. [36] developed a device that can be used for data 


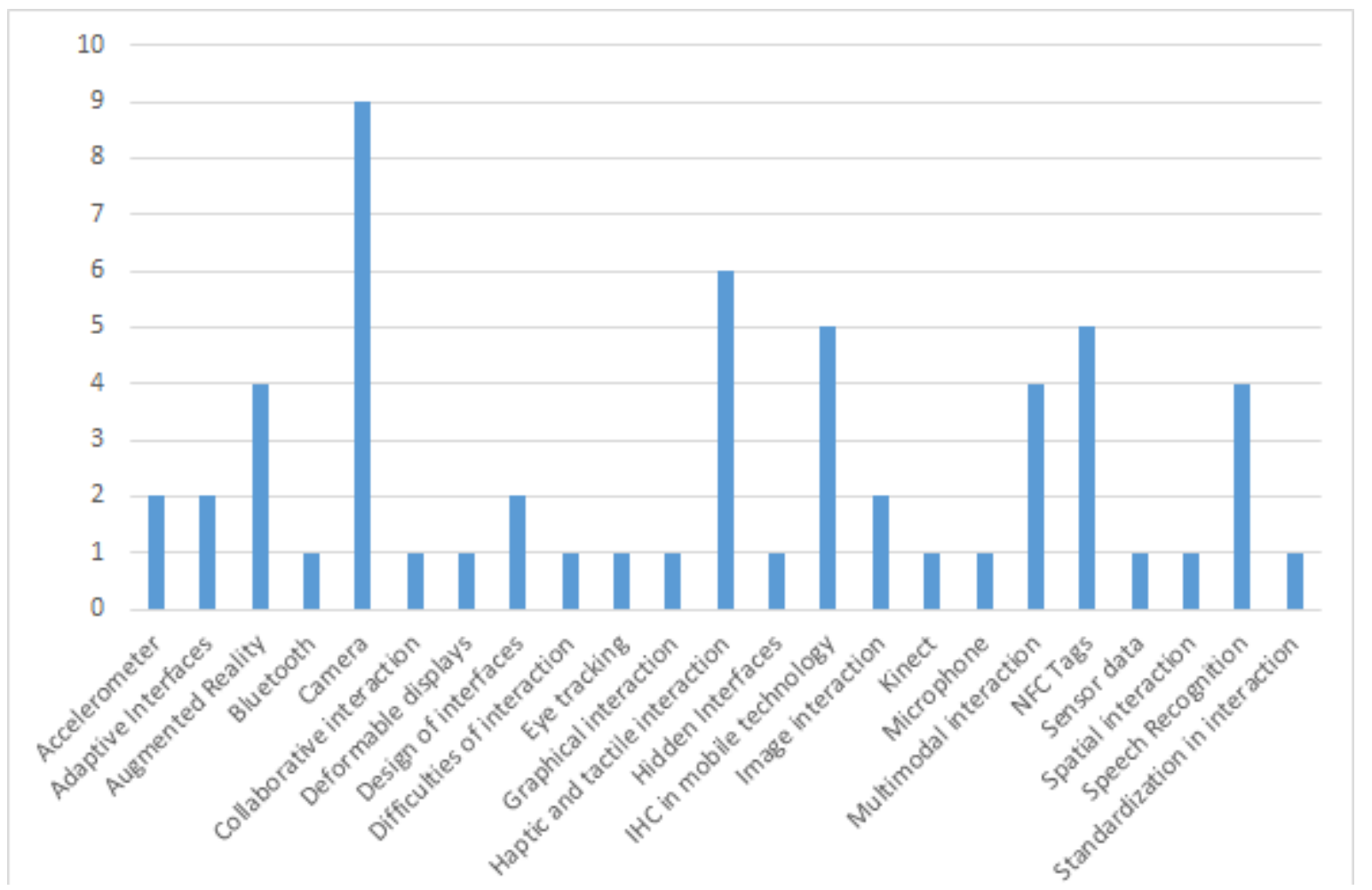

Fig. 4. Publications focused on interaction categorized by topic.

TABLE IX

EXAMPLES OF MOBILE COMPUTING INTERFACES

\begin{tabular}{|c|l|}
\hline 1 & $\begin{array}{l}\text { Portable physical feedback system that employs Bluetooth commu- } \\
\text { nication and OSC (Open Sound Control), allowing mouth-shape } \\
\text { recognition and sound-level analysis to generate and deliver the act } \\
\text { of blow as a sense of touch to the other party, generating an expanded } \\
\text { mode of interaction. [65] }\end{array}$ \\
\hline 2 & $\begin{array}{l}\text { Virtual interface trackpad that tracks user input on any surface near } \\
\text { the mobile device and extends the reach of the interaction over the } \\
\text { touchscreen, uses the sound source localization technique and adopts } \\
\text { the acoustic signal as the main means for interaction. [66] }\end{array}$ \\
\hline 3 & $\begin{array}{l}\text { Development of intelligent jewelry, which removes the disconnect } \\
\text { between the wearable and the screen and focuses on the positive } \\
\text { psychological, tactile and performative aspects. [67] }\end{array}$ \\
\hline 4 & $\begin{array}{l}\text { Design of an eye tracker to reduce the need for eye tracking detecting } \\
\text { and computing, estimating the look by using a small subset of pixels } \\
\text { per frame. [68] }\end{array}$ \\
\hline 5 & $\begin{array}{l}\text { Interface for mobile phone devices using Morse code by means of a } \\
\text { unique key as an adapted access communication tool, allowing users } \\
\text { with physical disabilities to be able to make / respond to phone calls } \\
\text { or send / receive SMS messages. [19] }\end{array}$ \\
\hline
\end{tabular}

acquisition during movement to estimate kinematics in humans with motor impairment; Jeet et al. [41] proposed a prototype system that can provide a hands-free remote control for people with quadriplegia who do not have to send verbal commands for the selection of home appliances; Kostikis et al. [55] describes a smartphone-based method for detecting and quantifying hand tremor associated with movement disorders using accelerometer signals and gyroscope embedded in the users's phone; Xia et al. [74] proposed the concept of multimodal vocal interaction "Voz-TouchVision" based on multi-touch interaction and corresponding visual graphics; Yamamoto et al. [63] has developed a speech-input-driven embodied interaction mobile phone with a Narikiri-headset to reflect users' head movements and actions directly in InterActor (character) by an acceleration sensor and a gyro sensor.

In this context, to manipulate data from several sensors used in mobile devices requires a structure for storing, merging and processing this data. Billen [78] proposed a structure like this considering GPS, light, accelerometer, gyroscope and orientation of mobile device. Some papers explored the use of sensors of devices for mobile interaction: accelerometer [69] [106] [26]; microphone [88]; camera [79] [98] [100] [60] [39] [40] [103]; Kinect depth sensor [61].

Some papers employed augmented reality on mobile devices, to design a serious game [70], to generate a new architecture [99], and to provide immersive experiences [97] [73]. This technology can be used to motivate and stimulate the use of AAC, or to aid in the learning of important concepts related to this theme merging virtual objects with real-world images. In addition, computer vision technology can recognize hands-free gestures from live images to allow intuitive interactions, such presented in Yang et al. [64].

Collaborative interaction, such as proposed in Kambona et al. [83] can also be explored to aid the knowledge acquisition or to stimulate the practice of AAC.

Interesting study opportunities have been identified, such as deformable displays [77] that can physically mutates to better 
TABLE $X$

STUDIES RELATED TO EDUCATION, TEACHING AND LEARNING

\begin{tabular}{|c|c|}
\hline $\begin{array}{l}\text { Skiada et al. } \\
\text { [71] }\end{array}$ & $\begin{array}{l}\text { presents a mobile application to promote learning and } \\
\text { help children to improve some fundamental skills, } \\
\text { such as reading comprehension, spelling, short term } \\
\text { memory and solving mathematical problems. }\end{array}$ \\
\hline $\begin{array}{l}\text { Bereznak et al. } \\
\text { [44] }\end{array}$ & $\begin{array}{l}\text { analyzes the acquisition of day-to-day skills of stu- } \\
\text { dents with ASD by using prompting video. }\end{array}$ \\
\hline Epp [46] & $\begin{array}{l}\text { presents a tool to support mobile assisted language } \\
\text { learning. }\end{array}$ \\
\hline $\begin{array}{l}\text { Guerrero et al. } \\
\text { [72] }\end{array}$ & $\begin{array}{l}\text { presents a collaborative learning activity and a mobile } \\
\text { software tool to support grammar teaching for primary } \\
\text { school students. }\end{array}$ \\
\hline Basit et al. [35] & $\begin{array}{l}\text { talk about learning of Quran and related subjects on } \\
\text { mobile devices, discussing the problems faced by blind } \\
\text { individuals in using such applications. }\end{array}$ \\
\hline $\begin{array}{l}\text { Ismaili et al. } \\
\text { [47] }\end{array}$ & $\begin{array}{l}\text { investigates the potential of using smartphones and } \\
\text { tablets as alternative learning tools for assistive tech- } \\
\text { nology devices in formal and informal learning envi- } \\
\text { ronments. }\end{array}$ \\
\hline Mehigan [69] & $\begin{array}{l}\text { talk about mobile learning system for assist in the } \\
\text { learning of blind and visually impaired people. }\end{array}$ \\
\hline $\begin{array}{l}\text { Salazar et al. } \\
{[70]}\end{array}$ & $\begin{array}{l}\text { presents a serious game designed to teaching concepts } \\
\text { about cybersecurity using augmented reality. }\end{array}$ \\
\hline Yang et al. [64] & talk about gesture interaction for learning. \\
\hline $\begin{array}{l}\text { Villamarin et } \\
\text { al. [58] }\end{array}$ & talk about learning of gesture language by deaf people. \\
\hline Jung et al. [20] & $\begin{array}{l}\text { uses a focus group with dyslexia and other specific } \\
\text { learning difficulties to design sets of user-defined ges- } \\
\text { tures to invoke commands on a smartphone device. }\end{array}$ \\
\hline $\begin{array}{l}\text { Kouroupetroglou } \\
\text { et al. [48] [49] }\end{array}$ & $\begin{array}{l}\text { presents research in the field of universal design for } \\
\text { learning, showing that mobile assistive technology ap- } \\
\text { plications should involve all students, including those } \\
\text { with disabilities, in collaborative learning, reasoning, } \\
\text { and problem-solving activities. }\end{array}$ \\
\hline $\begin{array}{l}\text { Schoen et al. } \\
\text { [53] }\end{array}$ & $\begin{array}{l}\text { evaluates the feasibility and acceptability of the } \\
\text { SpeechPrompts mobile application, developed to aid } \\
\text { in the treatment of prosody deficits in children with } \\
\text { ASD and other speech disorders. }\end{array}$ \\
\hline $\begin{array}{l}\text { Recha et al. } \\
\text { [57] }\end{array}$ & $\begin{array}{l}\text { describes an initiative to support children with ASD to } \\
\text { learn how to speech, and propose a machine translation } \\
\text { device for sign language. }\end{array}$ \\
\hline $\begin{array}{l}\text { Dekelver et al. } \\
\text { [45] }\end{array}$ & $\begin{array}{l}\text { analyzes intellectual and development disabilities cate- } \\
\text { gories and describes particularities of mobile software } \\
\text { design for each category. }\end{array}$ \\
\hline
\end{tabular}

represent the on-screen content; haptic and tactile Interaction [82] [91] [92] [33] [34] [62] with the aim of improving and enriching user interactions when the visual channel is blocked or restricted (for example, for blind or mentally disabled users) or helping to reduce the visual demand associated with the use of mobile applications, allowing the transmission of important information while users' hands and eyes are otherwise occupied; use of NFC tags for classification of NFC-based interaction techniques [76] [59], self-reporting for patients [42], or to controle a smart-home [18] [32] [22]; hidden Interfaces [87]; adaptive interfaces [84] [93]; speech recognition [102] [12] [74]; eye tracking [13].

Some papers presented studies related to design of interfaces for mobile devices, such as a study proposing six rules that should be considered in the design of mobile interfaces [80], and a study describing how to design applications for different cultures [101]. As mobile design exists in a global networked culture, it is important to adopt a cross-cultural perspective when designing technology in these contexts, understanding the shared psychology of mobile users.

Regarding the difficulty of interaction with mobile devices, a study evaluates and reports the difficulties of interaction of quadriplegic people with the touchscreen [31].

In order to standardize the interaction in mobile devices, one of the papers presents a concept of mobile-gesture, platformindependent notation, called Monox (MObile NOtation - eXtensible), which provides a common basis for collaborative design and interaction analysis mobile [81].

\section{DISCUSSION}

Based on the mapped studies, the research questions defined for this systematic mapping study were answered.

The main question in this study aimed at identifying the recent instruments available for practicing AAC via mobile devices and the possible modalities of interaction was answered. The mapping presented instruments that are used as assistive technology, either in AAC applications or for other purposes, as shown in Tab. V. Likewise, with respect to modalities of interaction and computational interfaces, it was possible to verify the wide variety of possibilities which are already being used, as shown in Fig. 4 and Tab. IX.

For each question presented in Table 1, an answer was elaborated based on the information extracted from the mapped studies. As regards to Question 1) What are the recent modalities of interaction used on mobile devices? There are currently many options for mobile interaction offering different degrees of accessibility. Among the interaction modalities identified are: augmented reality, multimodal interaction, speech recognition, look tracking, mobile device sensors, adaptive interfaces and attention/standardization in interface design. The human interaction with the world is inherently multimodal [115]. Thus, there is a growing effort by the scientific community to leverage human communication skills by means of speech, gestures, touch, facial expression and other modes of communication with interactive systems [116].

Considering that humans interact with the world mainly by means of their main senses (sight, hearing, touch, taste and smell), the goal of research in this area is to develop technology, interaction methods and interfaces to eliminate existing limitations by using these together for a more intuitive user interaction. The development of multimodal interaction between humans and computers tries to address problems such as the selection of gestures or emblems that have similar meaning in a world audience (due to the existence of several cultures), proposing a reduction in the number of misinterpretations by means of the integration of types of interaction. As reported by Fernandes et al. [117], multimodal interaction area gained special relevance with the appearance of low-cost body and gesture recognition / detection devices associated with video game consoles such as: EyeToy1 (Playstation), Wii Remote or Microsoft Kinect (Xbox). More recently, a diversity of console-independent devices are becoming readily available, which can be acquired by end users and connected to multiple processing devices, more independently of manufacturers, but 
also more specialized in certain aspects of interaction and reduced cost. Examples of such devices are presented in Fernandes et al. [117] and include Leap Motion or Parallax Si1143 which allow the identification of finger gestures using images taken by infrared cameras and the Myo bracelet, which identifies gestures by detecting electrical activity in the user arm muscles, a technique known as electromyography. Parallel to low-cost gesture interaction, virtual reality and augmented reality have experienced a resurgence by means of low-cost immersion monitors and augmented reality glasses. Very little is known about how students with special needs can use mobile devices with augmented reality, for example. According to Fecich [118] this is a fundamental research topic to be explored, because it brings awareness not only to the field of special education but also considers the development of the field of educational technology in research and in the elaboration of a study with this student population.

Considering the Question 2) What are the limitations of the existing studies aimed at practicing AAC by means of mobile devices in terms of interaction? People with disabilities often need support tools for AAC purposes in their daily lives and often the interaction with mobile devices is a big challenge. Most commercially available technology AAC is primarily designed to support transactional communication such as voice needs and desires ("I am thirsty"), and computing power is primarily used to allow physical access (e.g., control by the look) or to improve the voice (by means of the technology Text-to-Speech). There is little use of computing power to improve access to words and phrases for personal narrative, an essential part of social interaction. Word selection and phrase building, content storage and retrieval with all of their associated cognitive requirements are still left out. Although there are many digital products available for AAC, they are often expensive, inflexible, and difficult to use: training is required to configure and customize, making it difficult for responsible or caretakers to do so. It was identified that many options are currently available, but these are not used; even the application considered as an industrial leader is used by less than $5 \%$ of the people who could benefit from it [11]. The question remains unanswered: why, despite decades of development, these devices do not meet the needs of their target audience?

Concerning Question 3) Are there specific methodologies to stimulate AAC practice in mobile devices? What modes of interaction are employed? No study presenting a methodology for the practice of AAC in mobile devices was found. This result suggests a gap in literature and a demand of studies for this purpose, or even that the "methodology" nomenclature has not been used to define studies related to the definition of ways to conduct practices of AAC. Some studies focused on participatory design methods, such as the papers of Borges et al. [119] [120] [121] are related to this question, but were not included in this mapping because of differences in the keywords used by the authors. Such studies focus on the inclusion of stakeholders with disabilities in participatory design practices to conceive customized assistive technology.
As the papers suggest, participatory activities are promising for designing solutions to the practice of AAC.

Regarding Question 4) What types of computer interface are currently available to facilitate the interaction of users with mobile devices? Devices and alternative input methods are used to make computers accessible to users with compromised movements or other difficulties. Among the types of computational interfaces identified in the mapping are interfaces used to simulate the act of blowing, to extend the reach of the interaction on the touchscreen, intelligent jewelry, unique key to use Morse code, among others. Selecting an assistive interface requires maximizing the flow of information and minimizing the effort (physical and mental) to use it [122]. Current alternatives include non-invasive brain-computer interfaces, eye tracking, electromyography, sip-and-puff (blowing), voice commands, chin control, head control, mouth joystick and tongue control [123].

A possible perspective for the concept of accessibility is strongly related to the idea of Universal Design, which refers to making things as accessible as possible for a group of people as broad as possible. Regarding question 5) Are aspects of Human-Computer Interaction considered? How? Studies recognize that the development of systems and interfaces for assistive technology is particularly challenging from the IHC point of view. What would functionate for a general population cannot be assumed for some intended user groups [90]. It is particularly important to consider perspectives of users and their caregivers to develop something that functionate for them. Two of the most important requirements of a system to support the interaction between a disabled user and a mobile device are: flexibility and configurability to allow a fine personalization depending on the needs and conditions of the user. In addition, one of the main problems of smartphones is represented by their complex user interfaces, composed of many small icons and input methods that depend increasingly on keyboards via software, multitouch or gestures. These mechanisms are particularly heavy for users with perceptual, motor, or cognitive impairment who may not be able to select an area of the screen with sufficient accuracy or with the requested time. 8 of the mapped studies cite the application of specific HCI techniques, such as usercentered design methods; and user-centered communication (puts human nature and its needs at the center of the design, implementation, and evaluation of communication systems and technology). Usability is also cited in these publications, and some studies focus exclusively on this theme associated with mobile technology.

Finally, as regards to Question 6) What applications have been used as assistive technology to help people with communication difficulties? In addition to the AAC applications presented as a result of the publications read, there are some applications and devices used for AAC, cited in these publications, presented in Tab. VIII.

The mapping shows some points need to be better explored for the benefits of AAC, which can be useful in the context of universally accessible learning. For example: 1 . investigating 
pervasive computation associated with AAC; 2 . identify the reason why existing applications are not being used by people with disabilities; 3 . generate a methodology to help developers design effectively accessible AAC solutions; 4 . Generate a methodology that stimulates the practice of communication construction considering the user with communication problems or their relatives as central stakeholders; 5. employ computer vision techniques to make the use of communication boards more attractive. All of these actions can help people with communication disorders exercise their communicative abilities, influencing affective, emotional aspects and, consequently, contributing to their education and learning.

Based on the mapped studies, it was noticed that most of the existing solutions are employed and focused on specific situations and offer little flexibility and adaptability, essential characteristics for accessibility. The use of mobile devices carries less stigma than traditional AAC devices, but in return, demands greater care with interface design and forms of interaction. Thus, the multimodal interaction in adaptive interfaces, informed by IHC theories and good practices, seems to be a promising option for AAC applications to provide their users independence and competence in their communicative functions.

With respect to adaptability, an important point to be studied and that requires continuous improvement is related to the use of AAC systems by people who have, besides communication disorders, motor difficulties. Regardless of the origin of the motor problem, it is common these users to present abnormal postures and involuntary movements that sometimes may be uncontrollable, making the use of various interfaces unfeasible. Several studies have been developed to generate alternatives for these users to interact with computer systems. From the results of the mapping, it is possible to highlight the studies of Cakic et al. [36] and Kostikis et al. [55], which in different ways seek to analyze the movements made by people with motor disabilities. Other approaches use a combination of different technology, but it is still difficult to find interfaces that can be controlled by people with pathological movements, such as spasms or tremors. Since users with motor difficulties have involuntary movements, it is necessary to design processing algorithms to separate voluntary movements from involuntary movements. This means that it is essential to define the particularities of these users because they will not be able to control interfaces in any other way, even if the technology are very sophisticated.

\section{CONCLUSION}

This paper presented a systematic mapping on modalities of interaction in mobile devices associated with AAC, which resulted in the reading and categorization of 99 publications. The main objective was to provide an overview of what has been investigated in the context of this area. The results of this mapping can be useful in conducting research in the area of AAC, exploring the potentialities of mobile devices based on the theoretical and practical tools reported in the publications described. In this way, the aim is to help the AAC to effectively serve people with communication or interaction difficulties, whether in the school environment or in the performance of their daily activities, taking into account their individual needs. Among the main conclusions of the mapping, it has been identified that it may be useful develop a methodology or process to perform the practice of AAC in mobile devices exploring different modalities of interaction. There is a great potential in current technology to provide flexibility and adaptability in mobile devices, enabling information sharing and continuous learning. By strengthening human interactions, their capacities and cognitive abilities are expanded, allowing new and more elaborate learning, contributing to their social inclusion.

\section{ACKNOWLEDGMENT}

The authors acknowledge the financial support of CNPq by means of the so-called MCTI-SECIS / CNPq N 84/2013 Assistive Technology.

\section{REFERENCES}

[1] Fatemeh Bambaeeroo and Nasrin Shokrpour. The impact of the teachers non-verbal communication on success in teaching. Journal of advances in medical education \& professionalism, 5(2):51, 2017.

[2] Tania Rossi Garbin et al. Alternative communication environments based on augmented reality for children with cerebral palsy: a proposed curriculum in action. 2008.

[3] C. Gevarter, M. F. OReilly, L. Rojeski, N. Sammarco, R. Lang, G. E. Lancioni, and J. Sigafoos. Comparisons of intervention components within augmentative and alternative communication systems for individuals with developmental disabilities: A review of the literature. Research in Developmental Disabilities, 34(12):4404 - 4414, 2013.

[4] Simone Krüger and Ana Paula Berberian. Augmentative and alternative communication system (aac) for social inclusion of people with complex communication needs in the industry. Assistive Technology, 27(2):101-111, 2015.

[5] Sanz Cecilia, Guisen Andrea, Baldassarri Sandra, Marco Javier, Cerezo Eva, et al. Games as educational strategy: A case of tangible interaction for users of alternative and augmentative communication. In Collaboration Technologies and Systems (CTS), 2013 International Conference on, pages 377-381. IEEE, 2013.

[6] Meryl Alper. Augmentative, alternative, and assistive: Reimagining the history of mobile computing and disability. IEEE Annals of the History of Computing, 37(1):96-96, 2015.

[7] Kathleen Kangas and Lyle Lloyd. Early cognitive skills as prerequisites to augmentative and alternative communication use: What are we waiting for? Augmentative and Alternative Communication, 4(4):211221,1988

[8] Patrick Coe et al. Forcephone: new prototype with pressure and thermal feedback. 2015.

[9] Kewalin Angkananon, Mike Wald, and Lester Gilbert. Applying Technology Enhanced Interaction Framework to Accessible Mobile Learning. Procedia - Procedia Computer Science, 27(Dsai 2013):261270, 2014.

[10] Vanessa Tavares de Oliveira Barros, Cristiane Affonso de Almeida Zerbetto, Kátia Tavares Meserlian, Rodolfo Barros, Murilo Crivellari Camargo, and Táthia Cristina Passos de Carvalho. Barros - 2014 DayByDay Interactive and Customizable Use of Mobile, 2014.

[11] Karyn Moffatt, Golnoosh Pourshahid, and Ronald M. Baecker. Augmentative and alternative communication devices for aphasia: the emerging role of smart mobile devices. Universal Access in the Information Society, pages 1-14, 2015.

[12] Kwang B Lee. The Design and Development of User Interfaces for Voice Application in Mobile Devices. 2006.

[13] Robert Gabriel Lupu, Florina Ungureanu, and Valentin Siriteanu. Eye Tracking Mouse for Human Computer Interaction. 2013.

[14] Barbara Kitchenham. Procedure for undertaking systematic reviews. Computer Science Depart-ment, Keele University (TRISE-0401) and National ICT Australia Ltd (0400011T. 1), Joint Technical Report, 2004. 
[15] Elizabete Munzlinger, Fabricio Batista Narcizo, and José Eustáquio Rangel de Queiroz. Sistematização de revisões bibliográficas em pesquisas da área de IHC. (translated) Systematization of bibliographic reviews in HCI area surveys. In Companion Proceedings of the 11th Brazilian Symposium on Human Factors in Computing Systems, pages 51-54. Brazilian Computer Society, 2012.

[16] Cleyton VC Magalhães, Ronnie ES Santos, Fabio QB da Silva, and Alex Sandro Gomes. Characterizing research in informatics in education in brazil: a systematic mapping of sbie publications. In Brazilian Symposium on Computers in Education - SBIE), volume 24, page 22, 2013

[17] Kai Petersen, Sairam Vakkalanka, and Ludwik Kuzniarz. Guidelines for conducting systematic mapping studies in software engineering: An update. Information and Software Technology, 64:1-18, 2015.

[18] Juan Bautista Montalvá Colomer, María Fernanda Cabrera-umpiérrez, Silvia De Los Ríos Pérez, Miguel del Castrillo, Páramo, and María Teresa Arredondo Waldmeyer. Developing an Augmentative Mobile Communication System. pages 269-274, 2012.

[19] Cheng-Huei Yang, Hsiu-Chen Huang, Li-Yeh Chuang, and ChengHong Yang. A mobile communication aid system for persons with physical disabilities. Mathematical and computer modelling, 47(3):318-327, 2008.

[20] Yong Hee Jung and Shengeng Qin. User-defined gesture sets using a mobile device for people with communication difficulties. In Automation and Computing (ICAC), 2011 17th International Conference on, pages 34-39. IEEE, 2011.

[21] Rolf Black, Annalu Waller, Nava Tintarev, Ehud Reiter, and Joseph Reddington. A mobile phone based personal narrative system. In The proceedings of the 13th international ACM SIGACCESS conference on Computers and accessibility, pages 171-178. ACM, 2011.

[22] Naotsune Hosono, Yutaka Tomita, Hiromitsu Inoue, and Miwa Nakanishi. Urgent Mobile Tool for Hearing Impaired, Language Dysfunction. pages 413-416, 2014.

[23] Silvia Artoni, Maria Claudia Buzzi, Marina Buzzi, Claudia Fenili, Barbara Leporini, Simona Mencarini, and Caterina Senette. Designing a mobile application to record aba data. In International Conference on Computers for Handicapped Persons, pages 137-144. Springer, 2012.

[24] Kevin M Ayres, Sally B. Shepley, Karen H. Douglas, Collin Shepley, and Justin D. Lane. Ayres - 2016 - Mobile Technology as a Prosthesis Using Mobile Technology to Support Community Engagement and Independence, 2016.

[25] Lizeth Islas, Víctor M González, and Marcelo Mejía. Developing a Mobile Application for Language Disabled Children with User Centered Design. pages 236-240, 2013.

[26] Niranjan Uma Shankar. Assistive communication device for cognitive disability and spastic children. pages 137-140, 2011.

[27] Gennaro Cordasco, Marilena Esposito, Francesco Masucci, and Maria Teresa Riviello. Assessing Voice User Interfaces : The vAssist System Prototype. pages 91-96, 2014.

[28] Rosanna Yuen-yan Chan. Cloud Augmentative and Alternative Communication for People with Complex Communication Needs. pages 2727-2732, 2014.

[29] Jurica Babic, Ivan Slivar, Zeljka Car, and Vedran Podobnik. Prototypedriven Software Development Process for Augmentative and Alternative Communication Applications. In 3th International Conference on Telecommunications (ConTEL), pages 1-8, 2015.

[30] Firkhan Ali, Bin Hamid, and Said Mohamed. Development of Prototype Chat System Using Mobile Platform for Disable People. 57:33-39, 2012.

[31] Tiago Guerreiro, Hugo Nicolau, Joaquim Jorge, and Daniel Gonçalves. Assessing Mobile Touch Interfaces for Tetraplegics. pages 31-34, 2010.

[32] Elena de la Guía, Vicente López, Teresa Olivares, María D Lozano, Víctor Penichet, and Luis Orozco. Easy Smart-Home Environment to Assist Patients with Mobility Impairment. 2015.

[33] Huimin Qian. Improving Access to Mobile Technologies Using Tactile Feedback. (102):36-40, 2012.

[34] Eiji Aoki, Junji Hirooka, Nobuhiro Nagatomo, Toshihiko Osada, Hiroaki Nishino, and Kouichi Utsumya. Effects of Haptization on Disabled People. (092310005):1153-1157, 2010.

[35] Wafa Basit and Nuzhat Sultan. Easy learning of quran using mobile devices for blind and visually impaired. In Advances in Information Technology for the Holy Quran and Its Sciences (32519), 2013 Taibah University International Conference on, pages 155-158. IEEE, 2013.
[36] Nikola S Cakic and Mirjana B Popovi. Battery Operated Smart Device for Human Movement Measurement Based on Android OS Platform and Bluetooth Technology. 2014.

[37] Tanay Choudhary, Saurabh Kulkarni, and Pradyumna Reddy. A braille-based mobile communication and translation glove for deafblind people. In Pervasive Computing (ICPC), 2015 International Conference on, pages 1-4. IEEE, 2015.

[38] Massimiliano Donati, Luca Fanucci, Fabrizio Iacopetti, and Alessio Vecchio. A modular suite to support human-smartphone interaction for people with motor skill impairments. In Design \& Technology of Integrated Systems in Nanoscale Era (DTIS), 2015 10th International Conference on, pages 1-5. IEEE, 2015.

[39] Hanene Elleuch, Ali Wali, and Adel M Alimi. Smart Tablet Monitoring By a Real-time Head Movement And Eye Gestures Recognition System. 2014.

[40] Hanene Elleuch, Ali Wali, Anis Samett, and Adel M Alimi. A static hand gesture recognition system for real time mobile device monitoring. pages 195-200, 2015.

[41] Vikram Jeet, Hardeep Singh Dhillon, and Sandeep Bhatia. Radio Frequency Home Appliance Control based on Head Tracking and Voice Control for Disabled Person. pages 559-563, 2015.

[42] Andreas Prinz, Philipp Menschner, Matthias Altmann, and Jan Marco Leimeister. inSERT An NFC-based Self Reporting Questionnaire for Patients with impaired fine motor skills. pages 26-31, 2011.

[43] Anna N Lapyko, Li-Ping Tung, and Bao-Shuh Paul Lin. A cloudbased outdoor assistive navigation system for the blind and visually impaired. In Wireless and Mobile Networking Conference (WMNC), 2014 7th IFIP, pages 1-8. IEEE, 2014

[44] Sally Bereznak, Kevin M Ayres, Linda C Mechling, and Jennifer L Alexander. Video Self-Prompting and Mobile Technology to Increase Daily Living and Vocational Independence for Students with Autism Spectrum Disorders. pages 269-285, 2012.

[45] Jan Dekelver, Marina Kultsova, Olga Shabalina, Julia Borblik, Alexander Pidoprigora, and Roman Romanenko. Design of Mobile Applications for People with Intellectual Disabilities. Creativity in Intelligent Technologies and Data Science, (October 2016):823-836, 2015.

[46] Carrie Demmans Epp. Mobile Adaptive Communication Support for Vocabulary Acquisition. 1:173-175, 2014.

[47] Jalal Ismaili, El Houcine, and Ouazzani Ibrahimi. Mobile learning as alternative to assistive technology devices for special needs students. Education and Information Technologies, 2016.

[48] Georgios Kouroupetroglou, Spyridon Kousidis, and Paraskevi Riga. The mATHENA Inventory for Free Mobile Assistive Technology Applications. 2:519-527, 2015.

[49] Georgios Kouroupetroglou and Paraskevi Riga. A methodological approach for designing and developing web-based inventories of mobile Assistive Technology applications. Multimedia Tools and Applications, 2016.

[50] Marina Kultsova, Roman Romanenko, Irina Zhukova, Andrey Usov, Nikita Penskoy, Tatiana Potapova, Software Engineering, and Volgograd State Technical. Assistive mobile application for support of mobility and communication of people with IDD. (Idd), 2016.

[51] Hyowon Lee, Bree Westphal, Leong Wei Kiat, Ngai-man Cheung, and Teo Chor Guan. When Intuitive Multi-touch Tablet Is Not Enough : Mobile Gaming for Extreme Usability. page 8, 2016.

[52] Natacsha Raposo, Hélio Rios, David Lima, Bruno Gadelha, and Thais Castro. An application of mobility aids for the visually impaired. pages 180-189, 2014.

[53] Elizabeth Schoen, Simmons Rhea, and Paul Frederick. Brief Report : A Mobile Application to Treat Prosodic Deficits in Autism Spectrum Disorder and Other Communication Impairments : A Pilot Study. Journal of Autism and Developmental Disorders, 46(1):320-327, 2016.

[54] Stefan Goetze, Feifei Xiong, Jan Rennies, Thomas Rohdenburg, and Jens-e Appell. Hands-Free Telecommunication for Elderly Persons Suffering from Hearing Deficiencies. 2010.

[55] N Kostikis, Senior Member, M Arnaoutoglou, C Kotsavasiloglou, and S Baloyiannis. Towards Remote Evaluation of Movement Disorders via Smartphones. pages 5240-5243, 2011.

[56] Marc Kowtko. Using assistive technologies to improve lives of older adults and people with disabilities. In Systems, Applications and Technology Conference (LISAT), 2012 IEEE Long Island, pages 1-6. IEEE, 2012

[57] K. Rekha and B. Latha. Mobile translation system from speech language to hand motion language 2014 International Conference on 
Intelligent Computing Applications MOBILE TRANSLATION SYSTEM FROM SPEECH LANGUAGE TO HAND. pages 411-415, 2014

[58] Sindey Carolina Bernal Villamarin, Diego Alejandro Cantor Morales, Carlos Andrés Ávila Reyes, and Christian Albeiro Sánchez. Application Design Sign Language Colombian for Mobile Devices. 2016.

[59] Gregor Broll, Roman Graebsch, Maximilian Scherr, Sebastian Boring, Paul Holleis, and Matthias Wagner. Touch to Play Exploring TouchBased Mobile Interaction with Public Displays. pages 15-20, 2011.

[60] Fang-yu Chen, Chia-cheng Chao, Kanoksak Wattanachote, and KuanChing Li. Using Gesture Annotation to Achieve Real-Time Communication under MSNP. pages 1-5, 2011.

[61] Ye Gu, Ha Do, Yongsheng Ou, and Weihua Sheng. Human Gesture Recognition through a Kinect Sensor. pages 1379-1384, 2012.

[62] Oliver S Schneider and Karon E Maclean. Improvising Design with a Haptic Instrument Real-time Feedback. pages 327-332, 2014.

[63] Michiya Yamamoto, Kouzi Osaki, Shotaro Matsune, Tomio Watanabe, and A Interactor. An Embodied Entrainment Character Cell Phone by Speech and Head Motion Inputs. pages 298-303, 2010.

[64] Mau-tsuen Yang and Wan-che Liao. Computer-Assisted Culture Learning in an Online Augmented Reality Environment Based on Free-Hand Gesture Interaction. 7(2):107-117, 2014.

[65] Inkyung Choi. Blowu: physical feedback for seamless remote interaction in mobile. In SIGGRAPH Asia 2014 Emerging Technologies, page 2. ACM, 2014.

[66] Seungeun Chung and Injong Rhee. vtrack: virtual trackpad interface using mm-level sound source localization for mobile interaction. In Proceedings of the 2016 ACM International Joint Conference on Pervasive and Ubiquitous Computing: Adjunct, pages 41-44. ACM, 2016.

[67] Alexandra Ling Ju and Mirjana Spasojevic. Smart jewelry: The future of mobile user interfaces. In Proceedings of the 2015 Workshop on Future Mobile User Interfaces, pages 13-15. ACM, 2015.

[68] Addison Mayberry, Pan Hu, Benjamin Marlin, Christopher Salthouse, and Deepak Ganesan. ishadow: design of a wearable, real-time mobile gaze tracker. In Proceedings of the 12th annual international conference on Mobile systems, applications, and services, pages 82-94. ACM, 2014

[69] Tracey J Mehigan. Harnessing Accelerometer Technology for Inclusive Mobile Learning. pages 1-2, 2009.

[70] Mikel Salazar, José Gaviria, Carlos Laorden, and Pablo G Bringas. Enhancing Cybersecurity Learning through an Augmented Realitybased Serious Game Teaching \& Learning Experiences in Engineering Education. pages 602-607, 2013.

[71] Roxani Skiada, Eva Soroniati, Anna Gardeli, and Dimitrios Zissis. EasyLexia : A Mobile Application for Children with Learning Difficulties. Procedia - Procedia Computer Science, 27(Dsai 2013):218-228, 2014.

[72] Luis A Guerrero, Sergio Ochoa, and Cesar Collazos. A mobile learning tool for improving grammar skills. 2(2):1735-1739, 2010.

[73] Prashanth Bollam and Soumyajit Deb. Mobile Collaborative Augmented Reality with Real-time AR / VR switching. 2254(1):2254, 2015.

[74] Li Xia, Wang Xiaochun, Kang Kai, and Wang Dan. Research and Design of the "Voice-Touch-Vision " Multimodal Integrated Voice Interaction in the Mobile Phone. 2010.

[75] Átila V M Moreira, Vicente V Filho, and Geber L Ramalho. Understanding mobile game success : a study of features related to acquisition , retention and monetization. 5(2):2-13, 2014.

[76] Gregor Broll, Wolfgang Reithmeier, Paul Holleis, Matthias Wagner, and Docomo Euro-labs. Design and Evaluation of Techniques for Mobile Interaction with Dynamic NFC-Displays. pages 205-212, 2011.

[77] Jason Alexander. Interaction with Deformable Displays. pages 237 239, 2012.

[78] Nicolas Billen. A Mobile Sensor Data Acquisition and Evaluation Framework for Crowd Sourcing Data. pages 47-54, 2013.

[79] Shelley Buchinger, Ewald Hotop, Francesca De Simone, and Touradj Ebrahimi. Gesture and Touch Controlled Video Player Interface for Mobile Devices. pages 699-702, 2010.

[80] Paul Craig. Interactive Animated Mobile Information Visualisation. 2015.

[81] Roman Ganhör and Wolfgang Spreicer. Monox : Extensible Gesture Notation for Mobile Devices. pages 203-212, 2014.

[82] Jani Heikkinen and Thomas Olsson. Expectations for User Experience in Haptic Communication with Mobile Devices. 2009.
[83] Kennedy Kambona, Lode Hoste, Elisa Gonzalez Boix, and Wolfgang De Meuter. Coordinating Collaborative Interactions in Web-based Mobile Applications. pages 181-190, 2015.

[84] Shaun K Kane. Context-Enhanced Interaction Techniques for More Accessible Mobile Phones. (93):39-43, 2009.

[85] Joon-gyum Kim, Alvin Chiang, and Sung-ju Lee. A Picture is Worth a Thousand Words : Improving Mobile Messaging with Real-time Autonomous Image Suggestion. pages 51-56, 2016.

[86] Joon-gyum Kim and Sung-ju Lee. Demo : MilliCat : Real-Time Autonomous Image Suggestion for Mobile Messaging. 2016.

[87] Moon-hwan Lee, Da-hoon Kim, Hyun-jeong Kim, and Tek-jin Nam. Understanding Impacts of Hidden Interfaces on Mobile Phone User Experience. pages 45-48, 2012.

[88] Hong Lu, Wei Pan, Nicholas D Lane, Tanzeem Choudhury, and Andrew T Campbell. SoundSense : Scalable Sound Sensing for PeopleCentric Applications on Mobile Phones. pages 165-178, 2009.

[89] Zdenek Mikovec, Ladislav Cmolik, Jiri Kopsa, and Pavel Slavik. Beyond traditional interaction in a mobile environment : New approach to 3D scene rendering. 30:714-726, 2006.

[90] Cosmin Munteanu, Sharon Oviatt, Gerald Penn, and Randy Gomez. Designing Speech and Multimodal Interactions for Mobile, Wearable , and Pervasive Applications. pages 3612-3619, 2016.

[91] Young-woo Park. CheekTouch : An Affective Interaction Technique while Speaking on the Mobile Phone. pages 3241-3246, 2010.

[92] Huimin Qian, Ravi Kuber, and Andrew Sears. Towards developing perceivable tactile feedback for mobile devices. Journal of Human Computer Studies, 69(11):705-719, 2011.

[93] Tiago Alexandre Custódio Reis. Improving Mobile Interaction with Context-awareness, Multimodality, and Adaptive Interfaces. 5615(351):5615, 2010

[94] Tae Houn Song, Min Kyung Kim, and Jae Wook Jeon. Sensible Interface Using Multi-Sensors In Mobile Device. pages 637-641, 2009.

[95] Martin Spindler. Move Your Phone : Spatial Input-based Document Zoom \& Pan on Mobile Displays Revisited. pages 515-518, 2014.

[96] Genta Suzuki, Nobuyasu Yamaguchi, Shigeyoshi Nakamura, and Hirotaka Chiba. Mobile Interaction Using Steganographic Image on Mobile Display. pages 507-510, 2008.

[97] Ziying Tang and Xiaohu Guo. Real-Time 3D Interaction with Deformable Model on Mobile Devices. pages 1009-1012, 2011.

[98] Panu Vartiainen, Suresh Chande, and Kimmo Rämö. Mobile Visual Interaction Enhancing local communication and collaboration with visual interactions. 2006.

[99] Gabriela Tinti Vasselai, Paulo Cesar, and Rodacki Gomest. A Case Study of Augmented Reality for Mobile Platforms. 2(3):10-16, 2011.

[100] Chi Zhang, Joshua Tabor, Jialiang Zhang, and Xinyu Zhang. Extending Mobile Interaction Through Near-Field Visible Light Sensing. pages 345-357, 2015.

[101] Gustav Verhulsdonck. From Cultural Markers to Global Mobile : Using Interaction Design for Composing Mobile Designs in Global Contexts. Computers and Composition, 38:140-150, 2015.

[102] Arun Jagatheesan, Jong-hoon Ahnn, Thomas Phan, Abhishek Singh, and Juhan Lee. Hierarchical Automatic Speech Recognition Powered by Data Infrastructure. pages 1140-1141, 2014.

[103] Houssem Lahiani. Real Time Hand Gesture Recognition System for Android Devices. pages 591-596, 2015.

[104] Anna K Lekova and Maya I Dimitrova. Hand gestures recognition based on lightweight evolving fuzzy clustering method. In Image Information Processing (ICIIP), 2013 IEEE Second International Conference on, pages 505-510. IEEE, 2013.

[105] Zhang Linghao. A In-depth Consideration upon the Tendency of the Interaction Design of Smart Phone Interface. pages 0-4, 2010.

[106] Tea Marasovic and Vladan Papic. Accelerometer based gesture recognition system using distance metric learning for nearest neighbour classification tea. 2012.

[107] Oliver Schreer, Peter Eisert, Peter Kauff, Ralf Tanger, and Roman Englert. Towards robust intuitive vision-based user interfaces. In Multimedia and Expo, 2006 IEEE International Conference on, pages 69-72. IEEE, 2006.

[108] Kristina Lapin. Deriving Usability Goals for Mobile Applications. 2014.

[109] Benjamin Schooley, Steven Walczak, Neset Hikmet, and Nitin Patel. International Journal of Medical Informatics Impacts of mobile tablet computing on provider productivity, communications, and the process of care. International Journal of Medical Informatics, 88:62-70, 2016. 
[110] R Michael Barker, Sanae Akaba, Nancy C Brady, and Kathy ThiemannBourque. Support for aac use in preschool, and growth in language skills, for young children with developmental disabilities. Augmentative and Alternative Communication, 29(4):334-346, 2013.

[111] David Trembath, Susan Balandin, Leanne Togher, and Roger J Stancliffe. Peer-mediated teaching and augmentative and alternative communication for preschool-aged children with autism. Journal of Intellectual and Developmental Disability, 34(2):173-186, 2009.

[112] Susan Baxter, Pam Enderby, Philippa Evans, and Simon Judge. Barriers and facilitators to the use of high-technology augmentative and alternative communication devices: a systematic review and qualitative synthesis. International Journal of Language \& Communication Disorders, 47(2):115-129, 2012.

[113] Jasmina Ivšac Pavliša, Marta Ljubešić, and Ivana Jerečić. The use of aac with young children in croatia-from the speech and language pathologists view. In KES International Symposium on Agent and Multi-Agent Systems: Technologies and Applications, pages 221-230. Springer, 2012

[114] Janice Light and David McNaughton. Putting people first: Re-thinking the role of technology in augmentative and alternative communication intervention. Augmentative and Alternative Communication, 29(4):299-309, 2013.

[115] F. Quek, D. McNeill, R. Bryll, S. Duncan, X. Ma, C. Kirbas, K. E. McCullough, and R. Ansari. Multimodal human discourse: Gesture and speech. ACM Trans. Comput.-Hum. Interact., 9(3):171-193, September 2002.

[116] M. Turk. Multimodal interaction: A review. Pattern Recognition Letters, 36:189 - 195, 2014.

[117] L. Fernandes, R. R. Nunes, G. Matos, D. Azevedo, D. Pedrosa, H. Paredes, L. Barbosa, B. Fonseca, P. Martins, L. Morgado, B. Cardoso, and F. de Carvalho. Bringing user experience empirical data to gesturecontrol and somatic interaction in virtual reality videogames: an exploratory study with a multimodal interaction prototype. 2015.

[118] Samantha J Fecich. The use of augmented reality-enhanced reading books for vocabulary acquisition with students who are diagnosed with special needs. PhD thesis, The Pennsylvania State University, 2014.

[119] Luciana Correia Lima De Faria Borges, Lucia Vilela Leite Filgueiras, and Cristiano Maciel. Towards a participatory development technique of assistive technology for mobility and speech impaired patients. In Proceedings of the 10th Brazilian Symposium on Human Factors in Computing Systems and the 5th Latin American Conference on HumanComputer Interaction, pages 247-256. Brazilian Computer Society, 2011.

[120] Luciana Correia Lima de Faria Borges, Lucia Vilela Leite Filgueiras, Cristiano Maciel, and Vinicius Carvalho Pereira. Customizing a communication device for a child with cerebral palsy using participatory design practices: contributions towards the pd4cat method. In Proceedings of the 11th Brazilian Symposium on Human Factors in Computing Systems, pages 57-66. Brazilian Computer Society, 2012.

[121] Luciana Correia Lima de Faria Borges, Lucia Filgueiras, Cristiano Maciel, and Vinicius Pereira. A customized mobile application for a cerebral palsy user. In Proceedings of the 31st ACM international conference on Design of communication, pages 7-16. ACM, 2013.

[122] Julio Abascal. Users with disabilities: maximum control with minimum effort. Articulated Motion and Deformable Objects, pages 449-456, 2008.

[123] Marcelo Archajo Jose and Roseli de Deus Lopes. Human-computer interface controlled by the lip. IEEE journal of biomedical and health informatics, 19(1):302-308, 2015. 